\title{
Secure Data Sharing In Agriculture Using Blockchain Technology
}

\author{
${ }^{1}$ Anantraj I, ${ }^{2}$ Dr.Rajkumar N, ${ }^{3}$ Dr.S.Subhashini, ${ }^{4}$ Dr.B.Sumathy \\ ${ }^{1}$ Assistant Professor, Department of CSE, K. Ramakrishnan College of Technology, Trichy, Tamilnadu, India \\ ${ }^{2}$ Assistant Professor, Department of Computer Science, St. Claret College, Bengaluru, India \\ ${ }^{3}$ Associate Professor, Rathinam College of Arts and Science, Coimbatore, India \\ ${ }^{4}$ Associate Professor, Department of Instrumentation and Control Engineering, Srisairam Engineering College, \\ Chennai, India
}

Article History: Received: 10 January 2021; Revised: 12 February 2021; Accepted: 27 March 2021; Published online: 10 May 2021

\begin{abstract}
The utilization of information and data turns out to be progressively urgent for the farming area to improve profitability and manageability. Data and Communication Technology (ICT) significantly builds the viability and effectiveness of gathering, putting away, investigating and utilizing information in agribusiness. It permits agrarian experts and cultivating networks to handily acquire update-to-date data and accordingly settle on better choices in their every day cultivating. The proposed blockchain based horticulture web application assist ranchers with guaranteeing more prominent benefit through direct rancher to client correspondence. This application chips away at the premise of blockchain innovation, to stay away from the change information during transmission. This assistance helps business correspondence and acquires straightforwardness the framework. The horticultural data framework gives its clients to get data about, cultivating items and so forth Squares of time-stepped item subtleties are put away on all frameworks across a worth chain. The blockchain innovation can comply with the provenance of meals and alongside those traces makes dependable horticulture item deliver chains and assemble believe amongst ranchers and shoppers. As a confided in approach of setting away records, it really works with the usage of records pushed improvements to make cultivating extra intelligent.
\end{abstract}

\section{INTRODUCTION}

\section{BLOCKCHAIN TECHNOLOGY}

Blockchain expands on the possibility of P2P organizations and gives a general informational index that each entertainer can trust, despite the fact that they probably won't know or confide in one another. It gives a common and confided in record of exchanges, where permanent and encoded duplicates of data are put away on each hub in the organization. Monetary impetuses as local organization tokens are applied to make the organization deficiency lenient, and assault and plot safe. Blockchain and inferred innovations give a general and straightforward bookkeeping and administration layer for the Internet. All organization members have equivalent admittance to a similar information progressively. Exchanges running over the organization are straightforward to all entertainers and can be followed back to their starting point. Blockchain can likewise be depicted as a disseminated bookkeeping machine or a supranational administration machine that is public and straightforward. At the point when the organization approves an exchange by lion's share agreement, the exchange is for all time kept in touch with the blockchain. Something else, the exchange is dismissed and doesn't go through. Just exchanges that have been remembered for the blockchain are considered as legitimate and last. A Blockchain convention works on top of the Internet, on a P2P organization of PCs that all run the convention and hold an indistinguishable duplicate of the record of exchanges, empowering P2P esteem exchanges without a go between however machine agreement. Blockchain itself a document a common and public record of exchanges that records all exchanges from the beginning square (first square) until now. Blockchain is a common, trusted, public record of exchanges, that everybody can examine however which no single client controls. It is a dispersed information base that keeps a consistently developing rundown of exchange information records, cryptographically got from altering and modification. Blockchain has three unique sorts, for example public blockchain, private blockchain, and consortium blockchain. Bitcoin and Ethereum are the instances of public blockchain, anybody and from anyplace can go along with them and can get diminished at the hour of his will. This is sealed by the complex numerical capacities. The private blockchain is the inside open record of the organization and the joining on that blockchain is allowed by the organization possessing that blockchain. The square development and mining speed is much better in the private blockchain when contrasted with public blockchain because of the restricted hubs. The 2 consortium blockchain anyway exists among the organizations or gathering of organizations and rather than the agreement the standards of participations are assigned to oversee the blockchain exchanges all the more adequately. This exploration utilizes consortium blockchain as the blockchain is to be administered by a public expert in the country. Square is the essential segment of the blockchain. A square comprises of the header and the body, the body of the square contains the exchanges being kept in touch with the framework. The header of the square contains the data about the square that incorporates past hash, nonce worth and trouble, and the time stamp of the square and the exchanges. The length of the square is variable and considered to have been among1 to $8 \mathrm{MB}$ of size. The header of the square interestingly recognizes the square to be put. 


\section{LITERATURE REVIEW}

Fine-grained two-factor assurance component for information partaking in distributed storage Authors: Zuo, Cong, Jun Shao, Joseph K. Liu, Guiyi Wei, and Yun Ling. Propose another information security system for distributed storage, which holds the accompanying properties.

1) The cryptographic key is secured by the two elements. Just on the off chance that one of the two components works, the mystery of the cryptographic key is held.

2) The cryptographic key can be repudiated productively by incorporating the intermediary re-encryption and key partition strategies.

3) The information is secured in a fine-grained path by embracing the characteristic based encryption procedure. Cryptographic advantage supporting revocability is intermediary re-encryption (PRE) which was proposed. In an intermediary re-encryption conspire, an intermediary (e.g., the semi-confided in cloud) can change a ciphertext for a client into another ciphertext that another client can decode while the intermediary can adapt only the length of the ciphertext. PRE can be formalized into two classes regarding the heading of change: bidirectional and unidirectional. In bidirectional PRE, the intermediary can change the ciphertext from one client into another client and the other way around. In unidirectional PRE, the intermediary can just change over one way. To build the proficiency and security of PRE, numerous plans were proposed. The two-factor is acknowledged by isolating the mysterious key into two sections, one can be put away in a potential-uncertain spot, and the other is put away in an alter safe gadget. Just on the off chance that one of them is kept mystery, the proposition stays secure. Moreover, with the assistance of CPABE and PRE, acquired the fine-grained admittance control on encoded information and the revocability of alter safe gadget, separately

Bootstrapping a blockchain based environment for enormous information trade Authors: Chen, Jinchuan, and YunzhiXue. Propose a decentralized answer for large information trade. This arrangement targets developing an environment, inside which all participators can coordinate to trade information in a shared manner. The center piece of this arrangement is to use blockchain innovation to record exchange logs and other significant reports. Blockchain is a conveyed record kept up by all clients in a distributed manner. Along these lines we needn't bother with any outsiders. There will be various parts in the environment, for example information proprietor, information distributer, and clients and so forth Consequently the information proprietors don't have to give information administration. They simply pass their information to the information distributers, which are the expert information specialist co-ops. Besides, the logs of every exchange will be recorded in the blockchain, which is available to each client and can't be adjusted. Thusly the information proprietors and information distributers can review the utilization of their information. We will likewise talk about the procedures to additionally ensure information security, for example copyright and security. Proposed arrangement isn't to develop any business sectors. It contains three sections. Initially, propose an environment and clarify how various clients can participate together to make information trade, and how they profit by the biological system. Besides, the arrangement will incorporate a bunch of introductory specificatoins for bootstrapping the environment, including conventions of running the blockchain, necessities for administrations, and configuration of logs and so forth Additionally, the arrangement will give a few executions of the conventions and the business measures.

AI based protection saving reasonable information exchanging huge information market Authors: Zhao, Yanqi, Yong Yu, Yannan Li, Gang Han, and Xiaojiang Du. Propose another blockchain-based reasonable information exchanging convention huge information market. The proposed convention incorporates ring signature, twofold validation forestalling mark and similitude figuring out how to ensure the accessibility of exchanging information, security of information suppliers and reasonableness between information suppliers and information buyers. To begin with, the market director introduces the framework boundaries. At that point, the information supplier and the information shopper register with the market administrator as new clients. At the point when the information supplier registers available director, he needs to pay store to the market chief. Then, the information supplier distributes the subject records on the blockchain. The information customer look on the blockchain and solicitations information about specific points. The information supplier sends the encoded information to the information customer. From that point forward, the information buyer haphazardly challenges some information blocks and the information supplier reacts the moved substance to the information purchaser. At that point, the information purchaser assesses the substance and dynamic by exploiting the distance metric learning procedure. In the event that the information shopper reacts to buy the information, the information supplier sends the main DAPS mark to the information buyer. The information purchaser distributes unforeseen installment shrewd agreement to blockchain network. At long last, the information supplier calls shrewd agreement and sends the second signature of DPAS to blockchain to get the installment. The information buyer can separate the mysterious key by utilizing two DAPS marks to unscramble the ciphertext. In the event that the information customer can't decode all ciphertexts, he grumbles to the market chief. The director can uncover the personality of the information supplier and rebuff him by moving his store to the information purchaser. We expect the store is more than the genuine installment and the market chief doesn't connive with the information purchaser. In the 
framework, every element has his/her ECDSA key pair (PK, SK) and the SignSK(m) means the ECDSA signature on message $\mathrm{m}$.

Empowering character based trustworthiness reviewing and information offering to touchy data covering up for secure distributed storage Authors: Shen, Wenting, Jing Qin, Jia Yu, RongHao, and Jiankun Hu. Propose a distant information uprightness examining plan that acknowledges information offering to delicate data stowing away in this paper. In this plan, a sanitizer is utilized to disinfect the information blocks comparing to the delicate data of the record and changes these information squares' marks into legitimate ones for the cleaned document. These marks are utilized to check the uprightness of the disinfected record in the period of respectability reviewing. Therefore, our plan makes the record put away in the cloud ready to be shared and utilized by others relying on the prerequisite that the delicate data is covered up, while the distant information honesty reviewing is as yet ready to be productively executed. A sanitizer is utilized to clean the information blocks relating to the touchy data of the document. In our definite plan, right off the bat, the client blinds the information blocks relating to the individual touchy data of the first document and produces the comparing marks, and afterward sends them to a sanitizer. The sanitizer disinfects these dazed information blocks into a uniform configuration and furthermore cleans the information blocks comparing to the association's touchy data. It likewise changes the comparing marks into substantial ones for the cleaned document. This strategy understands the distant information honesty reviewing, yet additionally upholds the information sharing relying on the prerequisite that delicate data is secured in distributed storage. As far as we could possibly know, this is the principal conspire with the above capacities. In addition, our plan depends on personality based cryptography, which works on the intricate endorsement the executives. At last, the sanitizer sends this cleaned document and its comparing marks to the cloud. These marks are utilized to check the uprightness of the disinfected record in the period of honesty reviewing. At the point when the TPA needs to confirm the respectability of the cleaned document put away in the cloud, he sends an examining challenge to the cloud. And afterward, the cloud reacts to the TPA with a reviewing verification of information ownership. At long last, the TPA confirms the honesty of the disinfected record by checking if this inspecting evidence is right.

Security protecting ciphertext multi-sharing control for large information stockpiling Authors: Liang, Kaitai, Willy Susilo, and Joseph K. Liu. Propose a protection safeguarding ciphertext multi-sharing system to accomplish the above properties. It consolidates the benefits of intermediary re-encryption with mysterious method in which a ciphertext can be safely and restrictively shared on numerous occasions without releasing both the information on fundamental message and the personality data of ciphertext senders/beneficiaries. Here consider the situation where an intermediary intrigues with delegatee to bargain the hidden message and the mysterious key of delegator. Here, the insurance of the message is exceptionally hard to accomplish as the delegatee can generally decode the comparing ciphertext for the intermediary. The mysterious key of the delegator, notwithstanding, is feasible to be gotten. For the meaning of plot assaults model, we permit an enemy to obtain all reencryption keys, and the foe dominates the match in the event that it yields a legitimate mystery key of an uncorrupted client. We note that our definition is in the specific model where the foe needs to yield an objective character at the beginning of the game. With respect to the security model of namelessness, it is convoluted as in we order the game into two sub games: one is the secrecy for delegator (for example given the first ciphertext a foe can't yield the character of delegator), the other is the namelessness of re-encryption key (for example an enemy can't recognize a legitimate re-encryption key from an arbitrary one having a place with reencryption key space). This proposed work demonstrated the framework CCA-secure in the standard model under the decisional P-bilinear Diffie-Hellman presumption.

Accountability for information administration in cloud biological systems Authors: Felici, Massimo, Theofrastos Koulouris, and Siani Pearson. Present a reasonable model, comprising of qualities, practices and instruments for responsibility in the cloud. The proposed model permits us to clarify, as far as responsibility ascribes, cloudinterceded cooperations between entertainers. Cloud administrations are characterized regarding diverse fundamental qualities, administration models and organization models. Joining such highlights empowers diverse plans of action and cloud environments including different partners. Associations that utilization or give cloud administrations work in a perplexing unique climate, use cloud administrations inside a store network, and need to feel certain that suppliers further down that chain are responsible for how they oversee individual or potentially secret information. Responsibility is fundamental to a dependable cloud. Without responsibility, cloud shoppers will need certainty to put individual or potentially private information in the cloud. Changing to the cloud model includes changes in charge, in trust and security limits and, might be likewise, in lawful administrative prerequisites. To improve dependability of cloud biological systems, it is important to have a careful comprehension of the likely advantages and dangers of embracing the cloud [18]. Moving information to the cloud includes a move in duties across authoritative limits. This reallocation of obligations across the cloud biological system changes hazard basics (for example probability of event and seriousness) just as hazard view of such dangers. It gets important to comprehend weaknesses just as to recognize new instruments upgrading dependability of cloud environments. Responsible associations guarantee that commitments to secure information are noticed all through help supply-chains. Responsibility upgrades the certainty of specialist organizations, controllers and end clients to convey, use, and screen cloud administrations. It empowers cloud 
environments to situate themselves as for administrative systems. Responsibility gives a way to accomplishing consistence regarding administrative systems, empowering (straightforwardness, security and protection) components custom-made to ensure information and information subjects.

\section{EXISTING SYSTEM}

A traditional information exchanging biological system, for example, the worked on rendition introduced, normally includes three gatherings, in particular: dealer, information trade (i.e., some center individual) and purchaser.In particular, the vender sends the dataset to a believed recordschangedegree and units a rightpromoting cost.. The purchaser will choose an information result of interest and put in an online request, like other web based business exchanges. After accepting the purchaser's installment, the information trade stage will communicate the bought information to the purchaser, and pay the vender (in the wake of deducting the administration expenses or commission). In any case, on the off chance that the crude dataset can't be straightforwardly utilized by the purchaser, the purchaser should re-measure the dataset to get the necessary outcomes fulfilling the purchaser's need. Present another information exchanging environment to supplement the current exchanging biological system. All in all, we move from information facilitating/trade as-a-support of information preparing as-a-administration, where the purchaser is paying for the examination of the vender's dataset. Hence, the test of getting the dataset is currently diminished to the test in getting the information preparing. In particular, we assemble an Intels Software Guard Extensions (SGX)- based secure execution climate to ensure the information handling, the source information and the examination results. Likewise build a safe information exchanging environment (SDTE), utilizing blockchain to forestall single-point disappointment. The utilization of blockchain likewise permits us to guarantee that each exchange in SDTE is straightforward, and works with the recognition of any adjustment of the value-based data. SDTE has three key elements, specifically: purchaser, dealer and SDTP. A purchaser conveys at least one information investigation contracts on SDTP to dissect the dealer's crude information and gets the information examination results. The merchant is the information source, and benefits from selling access of the information for examination. SDTP is a protected blockchain for contract arrangement, prerequisites coordinating and contract execution; and comprises of confided in hubs (SGX upheld) and ordinary hubs. The purchaser conveys his/her information examination contracts on SDTP, discovers some merchant dependent on the information of interest, and tracks down SDTP's confided in hubs. At that point, the merchant sends the information to the believed hubs chose by the purchaser. Information examination will be performed on the confided in hubs, and the outcome will be utilized to accomplish an agreement on SDTPs contract. Just the execution results will be shipped off the purchaser.

\section{DISADVANTAGE}

- Dishonest information trade may conceivable to reserve and exchange the merchant's dataset. 18

- A deceptive purchaser may likewise exchange the bought (crude) dataset, without the first dealer's information and endorsement.

- Manual framework is sets aside more effort to keep up information in web based business application. • Existing framework is physically, So it increment the odds of blunders.

- Lots of the time devoured for each report age.

- Sensitive information (cost of the item) requirements to adjust security insurance and consistence management.

\section{PROPOSED SYSTEM}

The current interaction of horticulture item deal through on the web, taken care of by specialist organization (Intermediator). The significant disadvantage in the current framework is that there will be no contacts between the client and the ranchers. In current framework the client doesn't think about rancher item subtleties and where it will be deal. There is an opportunity of value adjustment of agribusiness item by intermediator. In proposed work, create web application to assist ranchers with guaranteeing more noteworthy benefit through direct rancher to client correspondence. This help supports business correspondence and gets straightforwardness the framework utilizing blockchain based web application created for ranchers. This application offers assume to the town ranchers who need to utilize this office and who need to figure out how could it be conceivable and how they can utilize e-cultivating to sell their items. This would help the ranchers to deal their item through web application with all the more productively based on traffic interest. With the assistance of blockchain innovation, the cost of the item is won't changed by the delegate. Ranchers and purchasers are conveying in a safe manner. In blockchain SHA 256 is carrying out to create hash for fixing the sum esteems. After that nobody can change the sum. The partaking hubs in the square chain framework are altogether equivalent. Regardless of whether a few hubs in the framework are assaulted and obliterated, it won't harm the whole square chain framework.

\section{ADVANTAGES}

- Automation of existing manual data frameworks. 
- The square chain ensures recognizability and non-degradability of data. $\cdot$ It diminishes the achievement pace of aggressors. Monitor every day data trade at the worker by the manager.

\section{System Architecture}

Programming engineering includes the general design of programming deliberation, by utilizing deterioration and sythesis, with style of engineering and quality credits. A product engineering configuration should adjust to the primary usefulness and execution prerequisites of the framework, additionally as fulfill the non-useful necessities like unwavering quality, versatility, compactness, and accessibility. Programming design should portray its gathering of segments, their associations, cooperations among them and organization arrangement, everything being equal. Frameworks configuration suggests a logical way to deal with the arranging of a framework. it will take a base up or big picture perspective, yet whichever way the strategy is methodical wherein it mulls over completely related factors of the framework that should be made-from the design, to the predetermined equipment and programming, right directly down to the data and the manner in which it voyages and changes all through its movement through the framework. Frameworks configuration at that point covers with examination, frameworks designing and frameworks engineering.

Blockchain may tackle data the executives issues progressively across the get together and give chains of farming items. As recently referenced, a blockchain gives a permanent and straightforward library of information. This vault addresses a more proficient, consistent, and simple to-utilize choice to watch data of the strategy and improve recognizability across the accessibility chain. Likewise, sending this innovation permits keeping exact records of information about the source of the items, creation date, and insights regarding the maker, cost of the product. Blockchain builds security and trustiness among parties, which can become important when

items.

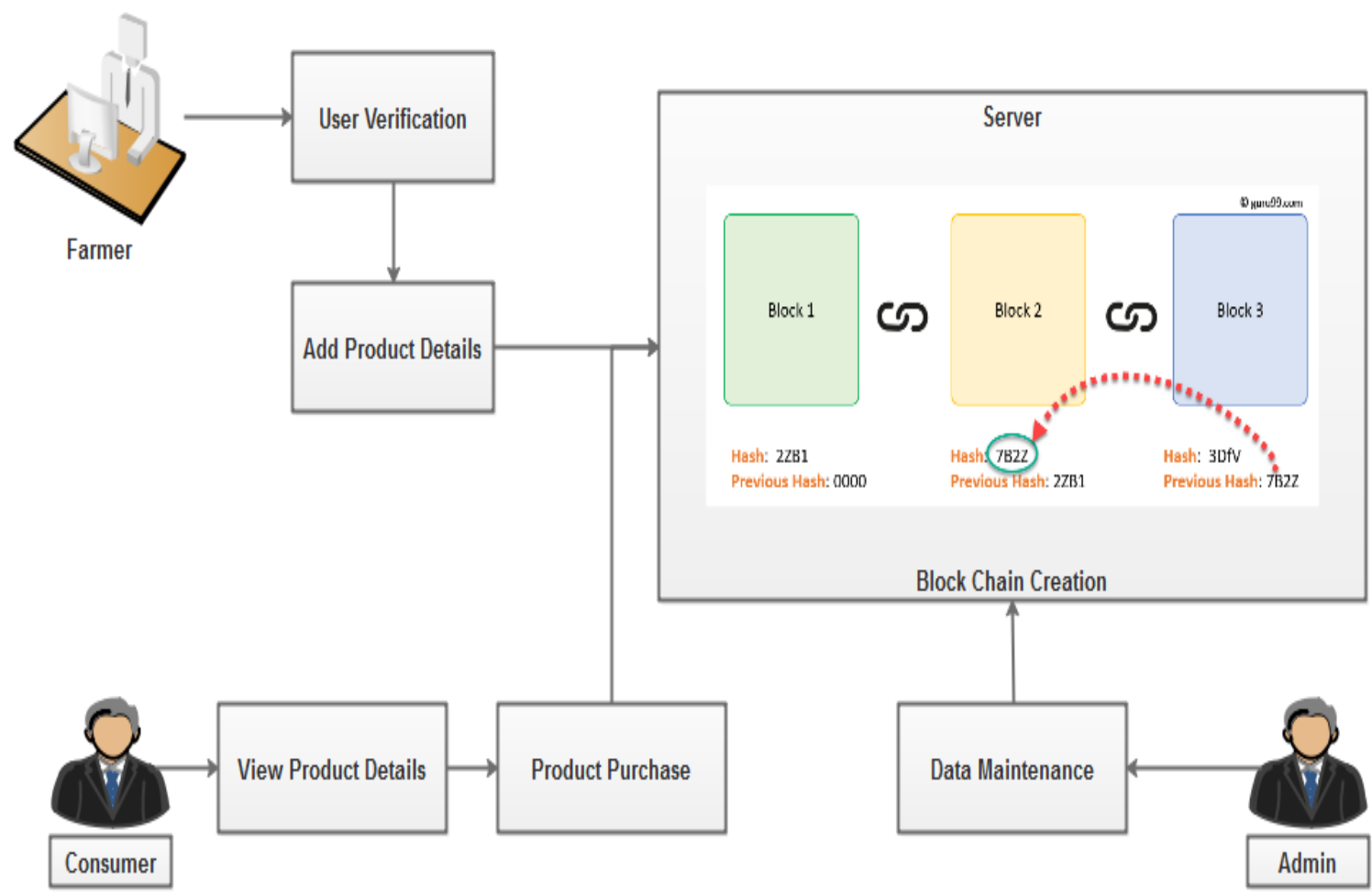

\section{SYSTEM IMPLEMENTATION}

\section{Modules:}

\section{Frameworkcreation}

Administrator make system that helps for ranchers upheld their prerequisites. Administrator has interesting username and secret phrase. Administrator has the obligation to deal with the all the important part. during this module administrator can see rancher subtleties. Enrolled rancher subtleties just saw by administrator. 


\section{UserEnrollment}

Client can login inside the framework utilizing username and secret key. Enlisted clients are just can login during this framework and make their cycle.

\section{Add Product details}

Subsequent to logging inside the entry the rancher will transfer his items into the data set which can enter his stock like all e-showcasing site, were the rancher will have the freedom to include refresh and erase any item from his stock.

\section{Blockchain creation}

The data of the square will be hashed utilizing the SHA-256 calculation. Each procedure block that affirms the fulfillment of the exchanges will have utilized the hash of past block, a substitution irregular number, and hash of the square to get the hash esteem which will be utilized by the procedure blocks. The fixing of the square methods the square has now been fixed with a hash work and subsequently the substance of the square can't be changed by guaranteeing the apparatus.

\section{Product Booking}

The client subsequent to signing in to the framework will look through result and may see the important part about the product that has the important part of the seller additionally in light of the fact that the accessible amount and cost of the product.

\section{Delivery status}

Conveyance status may be refreshed when the purchaser demand was fulfilled. Rancher can see the purchaser ask for and acknowledge the solicitation.

\section{ALGORITHM}

\section{Blockchain Technology}

Blockchain Technology A blockchain is an advanced idea to store information. These squares are fastened together, and this makes their information changeless. At the point when a square of information is anchored to different squares, its information can 23 never be changed again. It will be openly accessible to any individual who needs to see it until the end of time, in precisely the manner in which it was once added to the blockchain. The most embraced secure calculations related with the blockchain innovation are (SHA-1, SHA2, and SHA256) encryption on account of their extraordinary nature of hash work that makes special yields when given various information sources. The hash work here is a special key made to recognize an exchange that simultaneously distinguishes a person in the petrol production network. Blockchain innovation capacities are dependable for use in a hashing crypto technique, which makes a sufficient and solid hashing code and convert it from a touch of fixed size information to strings of character. Every exchange proposed in a blockchain are hashed together prior to pushing in a square, and the hash pointers associate each square to the following square for holding of past hash information as it is undisputable. Accordingly, any adjustments in the blockchain exchange of hashing capacity will bring about various hash line of character and influence every one of the elaborate squares.

\section{Block and Hash Generation}

1. A Block containing data about current exchanges.

2. Every information creates a hash.

3. A hash is a series of numbers and letters.

4. Exchanges are entered in the request in which they happened.

5. The hash depends on the exchange as well as the past exchange's hash.

6. Indeed, even a little change in an exchange makes a totally new hash.

7. The hubs check to ensure an exchange has not been changed by reviewing the hash.

8. Assuming an exchange is endorsed by a larger part of the hubs, it is composed into a square.

9. Each square alludes to the past block and together make the Blockchain. 
10. A Blockchain is successful as it is spread over numerous PCs, every one of which has a duplicate of the Blockchain

\section{EXPERIMENTAL RESULT AND EXPECTED OUTCOMES}

we move from information facilitating/trade as-a-support of information preparing as-a-administration, where the purchaser is paying for the examination of the dealer's dataset. Subsequently, the test of getting the dataset is currently diminished to the test in getting the information handling. In particular, we construct an Intels Software Guard Extensions (SGX)- based secure execution climate to ensure the information preparing, the source information and the investigation results. Additionally develop a safe information exchanging biological system (SDTE), utilizing blockchain to forestall single-point disappointment.

In current we give security to the client information and their item and we additionally confirm the client subtleties composer subtleties their item subtleties and we get the copy of the testament for checking if he is a rancher

\section{Data Set}

\section{For farmer registration:}

\begin{tabular}{ll} 
Name & - dfs \\
Father Name & - ghf \\
Gender & - Male \\
Age & -24 \\
Mobile & -9876453728 \\
Email & - dfs@ gmail.com \\
\hline Address & - dfs \\
User Name & - dfsg \\
Password &...- \\
Retype-password &...- \\
Certificate & - choose file
\end{tabular}

\section{New Worker Registration}

\begin{tabular}{ll} 
Name & -sam \\
Father Name & - mani \\
Gender & - Male \\
Age & -24 \\
Mobile & -9876453728 \\
Email & - sam@gmail.com \\
\hline Address & - sds \\
Amount & -589 \\
Work type & - choose from option
\end{tabular}

New Products can be uploaded by the farmers 


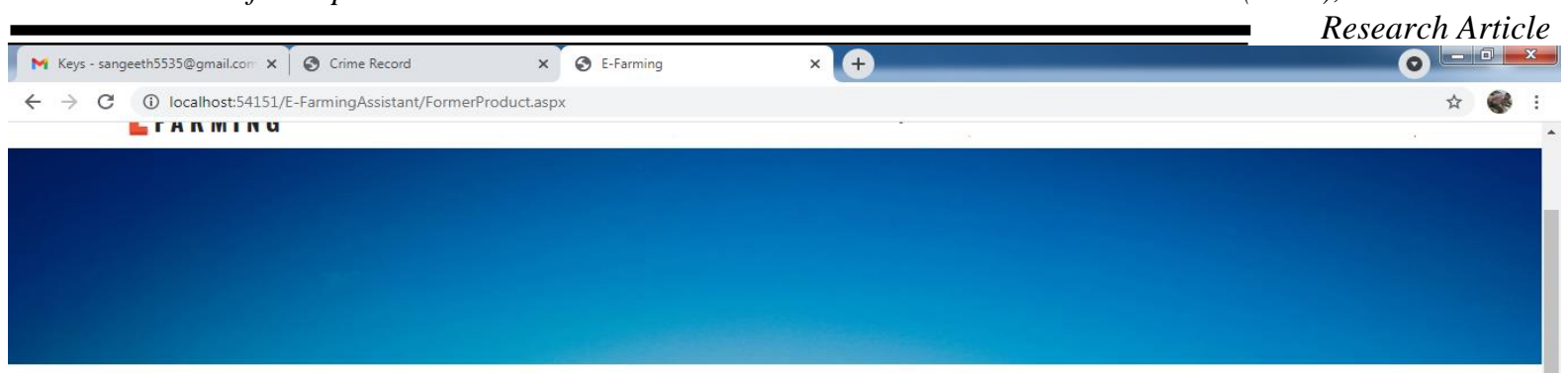

New Product Information

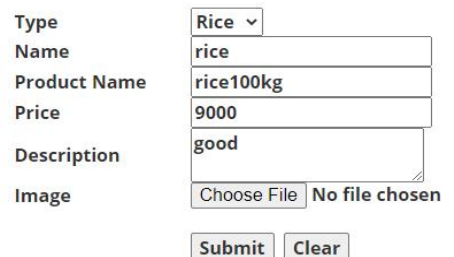

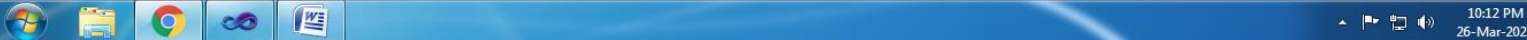

Customers can be able to view the details of the product

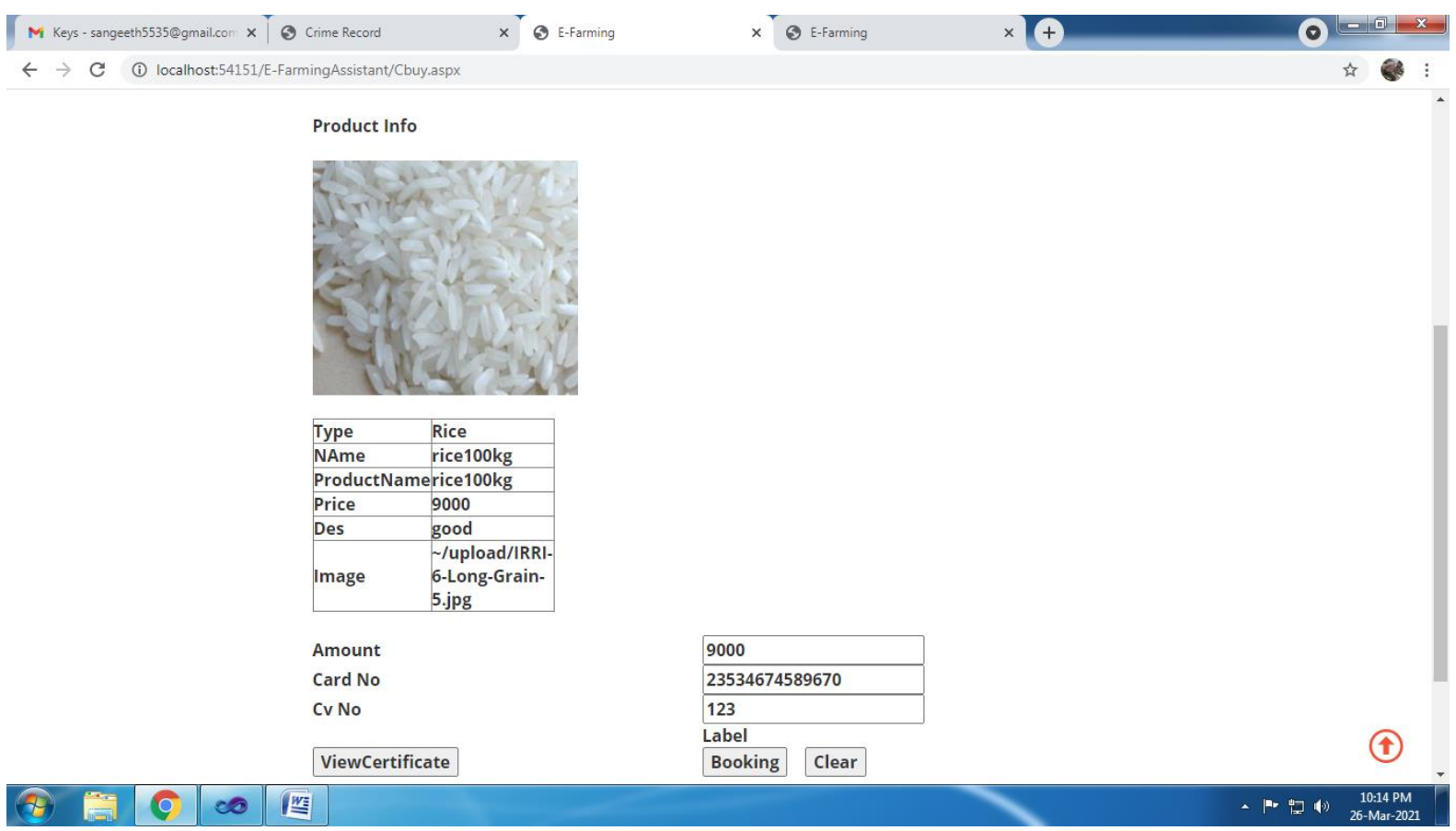

\section{Conclusion}

this content shows that blockchain innovation is utilized by numerous ventures and activities, having the opportunity to set up a demonstrated exchange charges, and work with continuous administration of production network exchanges during a safe and dependable way.ranchers and purchasers into the accessibility chain. Blockchain innovation offers numerous advantages, since it can give a protected, circulated on account of perform information exchanging among various untrusted parties. this is frequently a critical component in horticulture and product supply chains. to improve recognizability in esteem chains, a decentralized record assists with joining inputs, providers, makers, purchasers, controllers that are far separated, who are under 
various projects, various standards (strategies) or potentially utilizing various applications. Blockchain can possibly watch social and natural duty, improve provenance data, work with portable installments, credits and financing, decline

\section{Future Enhancement}

In future, incorporate different functionalities with this proposed framework like Notification and audit office for item ID and question the executives. Additionally furnish Online Payment with mastercard/open-end credit and so forth could even be coordinated into the framework to support ease of use and communications and believed climate to make a straightforward and more supportable horticulture item production network, incorporating

\section{REFERENCES}

[1] Zuo, Cong, Jun Shao, Joseph K. Liu, Guiyi Wei, and Yun Ling. "Fine-grained two-factor insurance instrument for information partaking in distributed storage." IEEE Transactions on Information Forensics and Security 13, no. 1 (2017): 186-196.

[2] Chen, Jinchuan, and YunzhiXue. "Bootstrapping a blockchain based biological system for enormous information trade." In 2017 IEEE worldwide congress on large information (bigdata congress), pp. 460-463. IEEE, 2017.

[3] Zhao, Yanqi, Yong Yu, Yannan Li, Gang Han, and Xiaojiang Du. "AI based protection saving reasonable information exchanging enormous information market." Information Sciences 478 (2019): 449-460.

[4] Shen, Wenting, Jing Qin, Jia Yu, RongHao, and Jiankun Hu. "Empowering character based respectability inspecting and information offering to delicate data covering up for secure distributed storage." IEEE Transactions on Information Forensics and Security 14, no. 2 (2018): 331-346.

[5] Liang, Kaitai, Willy Susilo, and Joseph K. Liu. "Protection safeguarding ciphertextmultisharing control for enormous information stockpiling." IEEE exchanges on data legal sciences and security 10, no. 8 (2015): 15781589 .

[6] Felici, Massimo, Theofrastos Koulouris, and SianiPearson."Responsibility for statistics management in cloud environments." In 2013 IEEE fifth International Conference on Cloud Computing Technology and Science, vol. 2, pp. 327-332. IEEE, 2013.

[7] Jung, Taeho, Xiang-Yang Li, Wenchao Huang, Jianwei Qian, Linlin Chen, Junze Han, JiahuiHou, and Cheng Su. "Accounttrade: "Accounttrade: Accountable conventions for substantial statistics replacing towards exploitative buyers." In IEEE INFOCOM 2017-IEEE Conference on Computer Communications, pp. 1-9. IEEE, 2017.

[8] Kamilaris, Andreas, Agusti Fonts, and Francesc X. Prenafeta-Boldv́. "'The ascent of blockchain innovation in farming and meals deliver chains. ." Trends in Food Science and Technology 91 (2019): 640-652.

[9] Shakhbulatov, Denisolt, Arshit Arora, Ziqian Dong, and Roberto Rojas-Cessa. "Blockchain execution for examination of carbon impression across food production network." In 2019 IEEE International Conference on (Blockchain), pp. 546-551. IEEE, 2019. 56

[10] Wu, Hsin-Te, and Chun-Wei Tsai. "An astute agribusiness network security framework dependent on private blockchains." Journal of Communications and Networks 21, no. 5 (2019): 503-508.

[11] Mohammadi, Mehdi, Ala Al-Fuqaha, SamehSorour, and Mohsen Guizani. "Profound gaining knowledge of for IoT big records and streaming examination: A review.." IEEE Communications Surveys and Tutorials 20, no. 4 (2018): 2923-2960.

[12] Lee, Sangho, Ming-Wei Shih, Prasun Gera, Taesoo Kim, Hyesoon Kim, and Marcus Peinado. "Inducing fine-grained control stream inside $\{$ SGX $\}$ areas with branch shadowing." In 26th \{USENIX\} security conference ( $\{$ USENIX\} security 17), pp. 557-574. 2017.

[13] Wu, Haiqin, Liangmin Wang, and Tao Jiang. "Secure and proficient k-closest neighbor question for area based administrations in re-appropriated conditions." Science China Information Sciences 61, no. 3 (2018): 1-3.

[14] Shinde, Shweta, Zheng Leong Chua, Viswesh Narayanan, and PrateekSaxena. "Forestalling page flaws from disclosing your mysteries." In Proceedings of the eleventh ACM on Asia Conference on Computer and Communications Security, pp. 317-328. 2016.

[15] Zhang, Fan, Ethan Cecchetti, Kyle Croman, Ari Juels, and Elaine Shi. "Local proclaimer: A validated information feed for shrewd agreements." In Proceedings of the 2016 aCMsIGSAC gathering on PC and interchanges security, pp. 270-282. 2016. 